\title{
Associação da empatia e do estresse ocupacional com o burnout em profissionais da atenção primária à saúde
}

\author{
Association of empathy and occupational stress with burnout \\ among primary health care professionals
}

João Paulo Pinheiro (https://orcid.org/0000-0001-8245-314X) ${ }^{1}$

Juliana Burges Sbicigo (https://orcid.org/0000-0001-9600-3318) ${ }^{1}$

Eduardo Remor (https://orcid.org/0000-0002-5393-8700) ${ }^{1}$

${ }^{1}$ Programa de PósGraduação em Psicologia, Universidade Federal do Rio Grande do Sul. R. Ramiro Barcelos 2600. 90035-003 Porto Alegre RS Brasil. joaopinheiro85@gmail.com.

\begin{abstract}
There are indications that burnout syndrome affects primary health care (PHC) professionals. The scope of this study was to investigate the association of empathy and occupational stress with the burnout of PHC professionals. A total of 348 workers filled out an an online assessment including occupational stress, empathy, and burnout scales. The empathetic-concern and perspective-taking empathy dimensions were negatively correlated with burnout $(p<.01)$, while personal anguish was positively related to it $(p<.01)$. The reward and effort occupational stress dimensions revealed greater weight in explaining the job disillusion and psychic exhaustion burnout subscales $(p<.001)$, respectively, while empathetic concern and personal anguish revealed the greatest weight for the explanation of indolence and guilt $(p<.001)$. Reward was the significant predictor of Profile 1 ( $p=.008$ ), the less severe form of the syndrome, while personal anguish $(p=.028)$ and effort ( $p=.012)$ revealed the same weight in the model for Profile 2, namely the most severe level of burnout. The results suggest interventions that focus on both work stressors and the empathy of the professionals involved.
\end{abstract}

Key words Occupational stress, Empathy, Burnout, Primary Healthcare
Resumo Há evidências de que a Síndrome de Burnout afeta profissionais da atenção primária à saúde (APS). Objetivou-se investigar a associação da empatia e do estresse ocupacional com o Burnout de profissionais da APS. 348 trabalhadores responderam a versões online de escalas de estresse ocupacional, empatia e Burnout. As dimensões de empatia preocupação empática e tomada de perspectiva correlacionaram-se negativamente com a sindrome ( $p<.01)$, enquanto angústia pessoal demonstrou relação positiva $(p<.01)$. As dimensões de estresse ocupacional recompensa e esforço apresentaram maior peso nos modelos das subescalas de Burnout ilusão pelo trabalho e desgaste psíquico $(p<.001)$, respectivamente, enquanto a preocupação empática e angústia pessoal demonstraram os maiores pesos para a explicação de indolência e culpa ( $p<.001)$. Recompensa foi o preditor significativo de Perfil 1 ( $p=.008$ ), forma menos grave $d a$ sindrome, enquanto angústia pessoal ( $p=.028)$ e esforço ( $p=.012)$ demonstraram o mesmo peso no modelo para Perfil 2, nível mais severo de Burnout. Os resultados sugerem intervenções que enfoquem tanto estressores do trabalho quanto a empatia dos profissionais.

Palavras-chave Estresse ocupacional, Empatia, Burnout, Atenção Primária à Saúde 


\section{Introdução}

A síndrome de Burnout consiste em uma consequência a estressores crônicos relacionados ao trabalho, em especial quando esse envolve diretamente a relação com pessoas ${ }^{1}$. A experiência da síndrome de Burnout pode ser identificada a partir do modelo de quatro dimensões de GilMonte ${ }^{2}$, integrado por ilusão pelo trabalho (percepção de que o trabalho proporciona desafios e o alcance de metas profissionais geram realização e satisfação pessoais; a ausência de ilusão pelo trabalho constituiria risco para Burnout), desgaste psíquico (sentimento de cansaço físico e emocional relacionado ao cotidiano laboral lidando com estressores e pessoas que apresentam algum tipo de problema), indolência (presença de atitudes negativas de indiferença, insensibilidade e distanciamento frente às pessoas atendidas, aos colegas e à organização) e culpa (corresponde ao surgimento de sentimentos de culpabilização por atitudes e comportamentos não condizentes com as normas internalizadas e com a cobrança social acerca do papel profissional).

Cabe esclarecer que, de acordo com Gil-Mon$\mathrm{te}^{2}$, o sentimento de culpa não se estabelece em todos os profissionais acometidos pelo Burnout. Por isso o modelo apresenta dois perfis da síndrome, sendo o Perfil 1 representado pelos indivíduos com baixos níveis de ilusão pelo trabalho, acompanhados de altos níveis de desgaste psíquico e indolência, com baixos níveis de culpa; enquanto níveis extremos das três primeiras dimensões acompanhados de altos níveis de culpa caracterizam o Perfil 2, a forma mais grave de Burnout ${ }^{2,3}$.

A literatura tem indicado associação da síndrome em profissionais de saúde com a exposição crônica a níveis elevados de estresse ocupacio$\mathrm{nal}^{4}$. Ambientes de trabalho são mais estressantes quando há alta carga laboral, pouco controle sobre o processo de trabalho e pouco suporte de chefia e colegas ${ }^{5}$, assim como quando ocorrem baixas recompensas pelos esforços do trabalhador ou comprometimento exagerado desse com sua função ${ }^{6}$, situações comuns no contexto da atenção primária à saúde brasileira ${ }^{7}$.

Todavia, além de fatores do contexto ocupacional, aspectos disposicionais, como o grau de empatia, também são considerados relevantes para explicar os níveis de Burnout encontrados entre profissionais de saúde ${ }^{8}$. Segundo Davis 9 , a empatia está representada pelas dimensões cognitivas: tomada de perspectiva (habilidade de espontaneamente adotar o ponto de vista psicológico do outro) e fantasia (capacidade de trans- por-se imaginativamente para os sentimentos e ações de personagens fictícios), e dimensões emocionais: preocupação empática (presença de sentimentos orientados ao outro de compaixão e preocupação) e angústia pessoal (sentimentos orientados para si de ansiedade e desconforto em situações interpessoais tensas).

De forma controversa, a literatura aponta que a empatia em profissionais de saúde pode estar tanto direta ${ }^{10}$ quanto inversamente ${ }^{11,12}$ relacionada à ocorrência de Burnout. Também foi proposto que essa relação possa variar de acordo com o tipo ou dimensão de empatia aferida ${ }^{13,14}$.Além disso, até o momento, não foram identificados estudos que englobassem concomitantemente o estresse ocupacional e a empatia dos profissionais na sua associação com o Burnout, comparando a sua relevância na compreensão da síndrome.

Sendo assim, o objetivo geral da presente pesquisa foi investigar a associação da empatia e do estresse ocupacional com o Burnout de profissionais da atenção primária em saúde. Como primeiro objetivo específico, o estudo buscou averiguar a relação das diferentes dimensões de empatia com o Burnout dos profissionais da APS. O segundo objetivo específico consistiu em comparar estresse ocupacional e empatia na sua associação com os níveis de Burnout apresentados por esses profissionais.

\section{Método}

\section{Participantes}

Participaram do estudo 348 profissionais das oito gerências distritais de saúde do município de Porto Alegre (RS, Brasil). Foram considerados aptos à participação todos os profissionais das categorias enfermeiro(a), técnico(a) de enfermagem, médico(a), cirurgião(ã) dentista, técnicos(as) e auxiliares de saúde bucal e agente comunitário(as) de saúde que atuassem na APS e que não estivessem afastados do trabalho por um período maior do que uma semana.

\section{Delineamento e procedimentos}

O estudo utilizou-se de delineamento transversal ${ }^{15}$. Antes do início da coleta de dados, foi realizada uma avaliação piloto para identificar a compreensão, formato e aceitação dos participantes em relação ao protocolo de avaliação utilizado. Participaram dessa etapa nove profissionais de saúde (seis agentes comunitários, dois 
enfermeiros e um médico) de uma mesma equipe de APS. Mediante entrevista individual, com o protocolo de avaliação online sendo apresentado em um tablet, os participantes do piloto puderam fazer sugestões em relação a cada questão em particular, assim como avaliar o protocolo de forma global, a partir de questões objetivas e abertas. A avaliação piloto deu indicações para a melhoria da apresentação do formulário online (ex. tamanho de fonte, ordenação de perguntas, modificação de opções de resposta, substituição de palavras, adição de termos, alteração de configuração de perguntas, esclarecimento do TCLE). Os resultados do teste piloto não foram incluidos na pesquisa e amostra final. Realizadas as alterações necessárias no protocolo de avaliação, a pesquisa foi divulgada nas gerências distritais, em reuniões com coordenadores de equipes de APS, quando foram obtidos seus contatos e foram distribuídos panfletos com os links de acesso da plataforma online Survey Monkey (https:// pt.surveymonkey.com/). Subsequentemente, a pesquisa foi divulgada pelas redes sociais e através dos coordenadores por correio eletrônico e aplicativo comunicador instantâneo (WhatsApp). Dessa forma, a amostragem foi não probabilística ${ }^{15}$. Ao acessar a plataforma, foi apresentada ao participante o Termo de Consentimento Livre e Esclarecido, seguido do protocolo de avaliação. A partir do recebimento do link para a coleta, os profissionais tiveram seis semanas para responder aos instrumentos, de julho a setembro de 2017. Nesse intervalo de tempo, foram enviados lembretes para participação por correio eletrônico e por aplicativo comunicador instantâneo.

\section{Variáveis e instrumentos}

Características sociodemográficas e ocupacionais - Questionário desenvolvido ad hoc para esta pesquisa com informações como sexo, idade, raça/cor (critério de classificação do $\mathrm{IBGE}^{16}$ ), categoria profissional e tipo de unidade em que atua [UBS, ESF, Unidade Mista (com equipe tradicional e ESF) ou Indígena].

Empatia - Avaliada a partir de itens do Interpersonal Reactivity Index (IRI), de Davis ${ }^{9}$, traduzido e validado no Brasil por Sampaio et al. ${ }^{17}$. A versão brasileira contém 26 itens em formato Likert de cinco pontos. Contudo, no presente estudo, houve uma falha no processo de coleta, fazendo com que os seis últimos itens da escala não fossem aplicados. Decidiu-se então por um processo de refatorialização com os 20 itens utilizados, seguindo as diretrizes de Izquierdo et al. ${ }^{18}$. Uma análise fatorial exploratória foi realizada, com o método de extração Fatoração de Eixo Principal (Principal Axis factoring) e o método de rotação Oblimin, $K M O=0.88$, Barlet Testp $<$ .001 , explicando $43,7 \%$ da variância observada. Identificou-se que os fatores obtidos apresentaram a maioria de itens alocados nos mesmos fatores da escala original, e que os dois itens que se deslocaram eram compatíveis em significado com o seu novo fator. Dessa forma, optou-se por manter as mesmas etiquetas da escala original para os quatro fatores obtidos. A subescala preocupação empática resultante $(\alpha=0.86)$ passou a ter oito itens $(3,5,6,10,11,12,16$ e 20$)$, angústia pessoal $(\alpha=0.67)$ apresentou quatro itens $(13,14$, 17 e 19), assim como fantasia ( $\alpha=0.65$, itens 1 , 2,15 e 18 ) e tomada de perspectiva ( $\alpha=0.73$, itens $4,7,8$ e 9). Como na versão original ${ }^{9}$, cada uma dessas subescalas constitui uma medida intervalar independente de empatia, não havendo escore geral da escala ou pontos de corte.

Estresse ocupacional - Avaliado a partir de duas escalas distintas:

a) Versão resumida da Job Stress Scale (JSS) ${ }^{19}$ adaptada para o português. A versão brasileira foi traduzida e validada por Alves et al. ${ }^{20}$. A escala possui 17 itens divididos em três subescalas. A primeira, demanda, com cinco itens (fidedignidade nesse estudo: $\alpha=0.73$ ), avalia pressões de natureza psicológica presentes no trabalho, tanto de caráter qualitativo quanto quantitativo. Controle, com seis itens $(\alpha=0.48)$, refere-se à possibilidade de o trabalhador usar suas habilidades intelectuais para desempenhar sua função, bem como ter autoridade sobre a forma de realizá-la. Apoio social, com seis itens $(\alpha=0.85)$, mensura os níveis de interação social com colegas e com a chefia. O instrumento é respondido através de escala Likert de quatro pontos. Nesse estudo, cada dimensão da escala foi considerada uma variável independente relacionada ao estresse ocupacional, conforme investigações anteriores usando o modelo demanda-controle ${ }^{21,22}$.

b) Versão Brasileira da escala Effort-reward Imbalance (ERI). A escala original, de Siegrist et al. ${ }^{23}$, foi traduzida e validada no Brasil por Chor et al. ${ }^{24}$. Possui 23 ítens divididos em três subescalas. A subescala esforço é composta de seis itens (fidedignidade nesse estudo: $\alpha=0.76$ ) e mede o esforço requerido do profissional frente a demandas do ambiente ocupacional, referente a aspectos qualitativos e quantitativos da carga de trabalho. A subescala recompensa $(\alpha=0.84)$ possui 11 itens e mede o quanto o profissional se sente recompensado pelo trabalho desempenha- 
do, em termos financeiros, de estima e em relação à ascensão e segurança no trabalho. Em ambas as subescalas, primeiramente os participantes precisam concordar ou discordar com o conteúdo de cada item. Ao concordar, devem atribuir uma pontuação em escala Likert de quatro pontos. A terceira subescala, overcommitment $(\alpha=0.55)$, mensura o empenho excessivo dos participantes em responder às demandas laborais, através de seis itens respondidos a partir de escala Likert de quatro pontos ${ }^{23}$. O escore geral de desequilíbrio esforço-recompensa pode ser calculado pela razão entre as pontuações obtidas nessas duas subescalas (e / ( $\mathrm{r} \times 0.454545)$ ), enquanto a subdimensão overcommitment é analisada como uma variável em separado ${ }^{23}$.

Burnout - Avaliado a partir do Cuestionario para la Evaluación del Síndrome de Quemarse por el Trabajo (CESQT) ${ }^{2}$. A versão brasileira utilizada foi validada por Gil-Monte et al. ${ }^{3}$. O instrumento possui 20 itens e quatro subescalas, respondidas através de escalas Likert de cinco pontos: ilusão pelo trabalho está representada por cinco itens (fidedignidade nesse estudo: $\alpha=0.89$ ), sendo invertidos para a pontuação total de Burnout. A subescala desgaste psíquico $(\alpha=0.86)$ possui quatro itens, indolência ( $\alpha=0.78)$ possui seis itens, e culpa $(\alpha=0.84)$ apresenta de cinco itens. A pontuação total da escala é obtida a partir da soma das pontuações das três primeiras dimensões, na seguinte fórmula: 20 - It $+\mathrm{Dp}+\mathrm{In}$. Os valores médios de resposta por item da pontuação total e da escala culpa podem ser utilizados para comparação com os escores da amostra de referência para profissionais de saúde obtida por Gil-Monte $^{2}$. Pode ser considerado em risco de Perfil 1 de Burnout o respondente cuja média por item da pontuação total apresenta-se igual ou superior a 1.93 (escore equivalente ao percentil $90 \mathrm{da}$ amostra de referência) e a média por item na subescala culpa apresenta-se menor do que 1.80 (percentil 90 na amostra de referência). O Perfil 2 também caracteriza-se pela média da pontuação total igual ou superior a 1.93 , porém acompanhada de pontuação média igual ou superior a 1.80 para a subescala culpa. Métodos semelhantes de pontuação foram utilizados em estudos prévios realizados com o CESQT no Brasil ${ }^{25,26}$ e no Uruguai ${ }^{27}$.

\section{Considerações éticas}

O presente estudo seguiu as diretrizes da Resolução $\mathrm{n}^{\circ} 466$, de 12 de dezembro de 2012, para regulamentação ética em pesquisas com seres humanos. $\mathrm{O}$ projeto foi apresentado à gestão do município, obtendo sua anuência para a implementação, e foi também submetido ao Comitê de Ética em Pesquisa do Instituto de Psicologia da UFRGS e ao Comitê de Ética em Pesquisa da Secretaria Municipal de Saúde do Rio Grande do Sul. Os participantes foram informados sobre os objetivos do estudo e a forma de coleta de dados, assim como sobre seus direitos de integridade física, emocional e mental. Eles foram também comunicados de que suas informações pessoais permaneceriam confidenciais, utilizados somente para análises estatísticas. Os dados individuais foram armazenados pela plataforma de pesquisa online Survey Monkey ${ }^{\circledR}$ (https://pt.surveymonkey.com/), ficando disponíveis apenas para os pesquisadores. O Termo de Consentimento Livre e Esclarecido conteve informações sobre o estudo e direito de evasão dos participantes a qualquer momento.

\section{Análise dos dados}

As análises foram realizadas com o software SPSS versão 20 (SPSS, Inc. Chicago, IL, USA). Os dados obtidos foram averiguados com relação ao grau de dispersão ou variabilidade (por exemplo, curtose e assimetria) para uma distribuição normal e para identificar a existência de escores extremos ou atípicos devido a erro do participante ao completar o questionário. Após esta inspeção inicial, foram calculadas as estatísticas descritivas de todas as variáveis: média, desvio padrão, frequência absoluta e relativa ${ }^{28}$.

Com a finalidade de responder ao primeiro objetivo específico do estudo, averiguar a relação das diferentes dimensões de empatia com o Burnout dos profissionais da APS, foram rodadas correlações de Pearson entre os escores das subescalas de empatia e de Burnout. Para averiguar a comparação entre estresse ocupacional e empatia na sua associação com os níveis de Burnout apresentados pelos profissionais da APS, o segundo objetivo específico da presente investigação, quatro modelos de regressão linear e dois de regressão logística foram rodados tendo como variáveis dependentes, respectivamente, cada uma das quatro dimensões de Burnout e a ocorrência de Perfil 1 ou Perfil 2 da síndrome.

As variáveis foram inseridas nos modelos através do método Enter, sendo incluídas nas regressões lineares aquelas que demonstraram correlações de Pearson com as variáveis dependentes. Foram selecionadas para as regressões logísticas aquelas variáveis que apresentaram associação com as categorias de desfecho em Testes 
t. Foram excluídos do modelo para ocorrência de Perfil 1 os participantes que demonstraram Perfil 2 de Burnout. No modelo para Perfil 2, foram incluídos somente aqueles que demonstraram algum dos perfis da síndrome. Dessa forma, foi possível investigar em separado os preditores potencialmente associados à transição entre a ausência de risco de Burnout e a ocorrência de sua forma menos grave, assim como averiguar as variáveis que podem estar relacionadas especificamente à transição do nível mais ameno da síndrome (Perfil 1) para o patamar mais grave de acometimento do Burnout (Perfil 2).

\section{Resultados}

Na Tabela 1, é apresentada a caracterização sociodemográfica e ocupacional da amostra total e por categoria profissional. Participaram do estudo 305 mulheres $(87.6 \%)$ e 39 homens (11.2\%). Os participantes foram majoritariamente pessoas da cor branca $[n=269(77.3 \%)]$. A média de idade foi de 40 anos (DP =9.7) e predominaram os profissionais enfermeiros [ $\mathrm{n}=102(29,3 \%)] \mathrm{e}$ atuantes no ESF $[\mathrm{n}=210(60.3 \%)]$.

\section{Correlações entre as dimensões de empatia e de Burnout}

A Tabela 2 apresenta as correlações entre as dimensões de Burnout, as dimensões de empatia, de estresse ocupacional e a idade dos participantes. As correlações entre empatia e Burnout foram todas fracas $\left(\mathrm{r}<.40^{28}\right)$. A preocupação empática esteve diretamente relacionada à ilusão pelo trabalho $(r=0.29, p<.01)$ e inversamente relacionada à indolência $(r=-0.25, p<.01)$. Angústia pessoal apresentou correlação positiva com desgaste psíquico $(r=0.29, p<.01)$, indolência $(r=$ $0.26, p<.01)$ e culpa $(r=0.33, p<.01)$. Fantasia demonstrou correlações diretas com ilusão pelo trabalho $(r=0.13, p<.05)$, desgaste psíquico $(r=$ $0.11, p<.05)$ e culpa $(r=0.16, p<.05)$. Por fim, assim como a preocupação empática, a tomada de perspectiva mostrou-se positivamente corrrelacionada à ilusão pelo trabalho $(r=0.21, p<$. $01)$ e negativamente relacionada à indolência $(r$ $=0.2, p<.01)$.

Tabela 1. Características Sociodemográficas e Ocupacionais da Amostra Total e por Categoria Profissional.

\begin{tabular}{|c|c|c|c|c|c|c|c|}
\hline & $\begin{array}{c}\text { Total } \\
348 \\
\end{array}$ & $\begin{array}{c}\text { Enferm. } \\
102(29,3)\end{array}$ & $\begin{array}{c}\text { Méd. } \\
38(10,9)\end{array}$ & $\begin{array}{c}\text { ACS } \\
75(21,6)\end{array}$ & $\begin{array}{l}\text { T/a. Enf. } \\
69(19,8)\end{array}$ & $\begin{array}{l}\text { Cir. Dent. } \\
39(11,2) \text {. }\end{array}$ & $\begin{array}{l}\text { T/ S. B. } \\
25(7,2)\end{array}$ \\
\hline & n (\%) & n (\%) & n (\%) & n (\%) & n (\%) & n (\%) & n (\%) \\
\hline \multicolumn{8}{|l|}{ Sexo } \\
\hline Mulheres & 305 (87.6) & $89(87.3)$ & $26(68.4)$ & $67(89.3)$ & $65(94.2)$ & 37 (94.9) & $25(100)$ \\
\hline Homens & $39(11.2)$ & $13(12.7)$ & $12(31.6)$ & $8(10.7)$ & $4(5.8)$ & $2(5.1)$ & $0(0)$ \\
\hline $\begin{array}{l}\text { Idade (M } \\
\text { (DP)) }\end{array}$ & $40.00(9.7)$ & $35.93(7.04)$ & $41.53(10.55)$ & $44.29(10.58)$ & $42.46(9.57)$ & $36.54(8.89)$ & $39.32(8.71)$ \\
\hline \multicolumn{8}{|l|}{ Raça/cor } \\
\hline Preto & $29(8.3)$ & $5(4.9)$ & $0(0)$ & $6(8.0)$ & $13(18.8)$ & $2(5.1)$ & $3(12.0)$ \\
\hline Pardo & $49(14.1)$ & $10(9.8)$ & $2(5.3)$ & $21(28.0)$ & $12(17.4)$ & $2(5.1)$ & $2(8.0)$ \\
\hline Branco & $269(77.3)$ & $87(83.3)$ & $36(94.7)$ & $47(62.7)$ & $44(63.8)$ & $35(89.7)$ & $20(80.0)$ \\
\hline Indígena & $1(0.3)$ & $0(0)$ & $0(0)$ & $1(1.3)$ & $0(0)$ & $0(0)$ & $0(0)$ \\
\hline \multicolumn{8}{|l|}{ Tipo US } \\
\hline UBS & $33(9.5)$ & $12(11.8)$ & $5(13.2)$ & $1(1.3)$ & $13(18.8)$ & $0(0)$ & $2(8.0)$ \\
\hline ESF & $210(60.3)$ & $67(65.7)$ & $17(44.7)$ & $55(73.3)$ & $36(52.2)$ & $21(53.8)$ & $14(56.0)$ \\
\hline Mista & $101(29.0)$ & $22(21.6)$ & $16(42.1)$ & $19(25.3)$ & $18(26.1)$ & $17(43.6)$ & $9(36.0)$ \\
\hline Indígena & $4(1.1)$ & $1(1.0)$ & $0(0)$ & $0(0)$ & $2(2.9)$ & $1(2.6)$ & $0(0)$ \\
\hline Perfil 1 & $50(14.4)$ & $14(13.7)$ & $2(5.3)$ & $15(20)$ & $6(8.7)$ & 7 (17.9) & $6(24)$ \\
\hline Perfil 2 & $155(44.5)$ & $45(44.1)$ & $18(47.4)$ & $31(41.3)$ & $36(52.2)$ & $15(38.5)$ & $10(40)$ \\
\hline $\begin{array}{l}\text { Nível crítico } \\
\text { de Burnout } \\
(\mathrm{P} 1+\mathrm{P} 2)\end{array}$ & $205(58.9)$ & $59(57.8)$ & $20(52.6)$ & $46(61.3)$ & $42(60.9)$ & $22(56.4)$ & $16(64)$ \\
\hline
\end{tabular}

Nota:\$: $\mathrm{n}=312$. Enfer. = Enfermeiro, Méd. = Médico, ACS = Agente Comunitário de Saúde, Téc./aux. Enf. = Técnico ou auxiliar de enfermagem, Cir. Dent. = Cirurgião Dentista, Téc./aux. S. B. = Técnico ou Auxiliar em Saúde Bucal, Tipo US = tipo de unidade de saúde à qual o profissional está vinculado, Mista = unidade de saúde que abriga tanto equipe tradicional quanto ESF. 


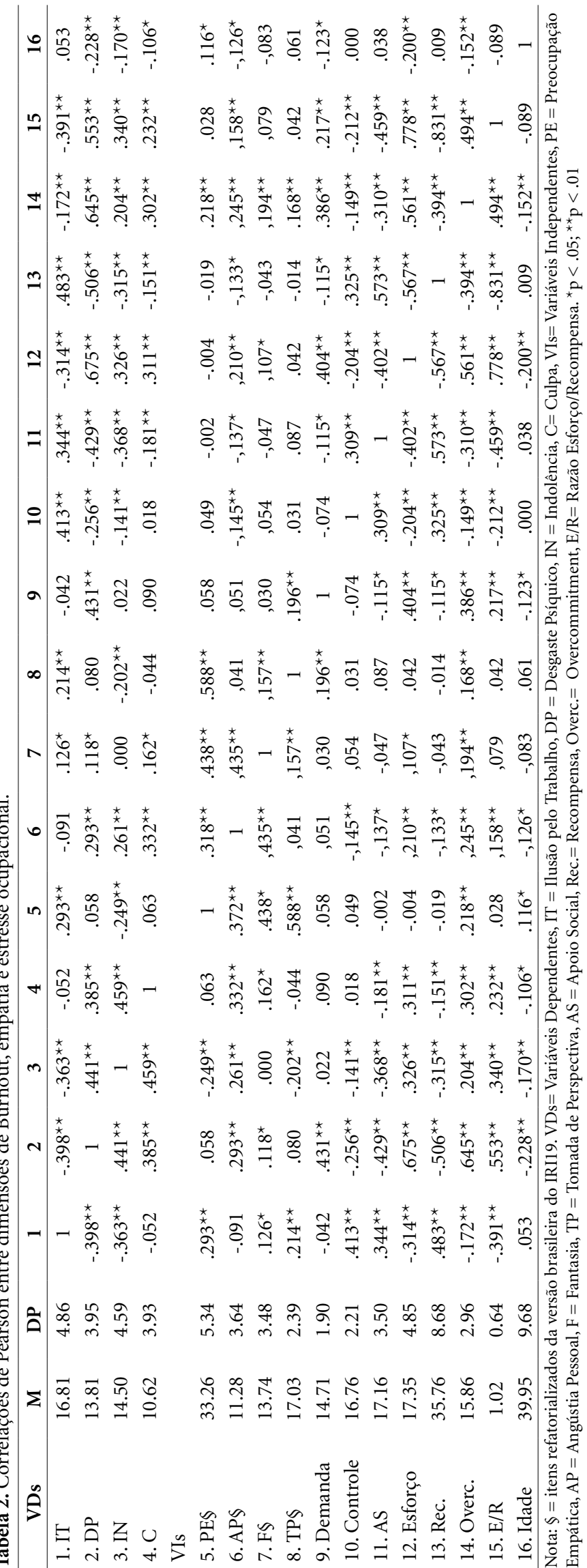

\section{Predição das dimensões de Burnout e da ocorrência de Perfil 1 e Perfil 2 da síndrome}

Foram incluídas nos modelos de regressão linear (Tabela 3) apenas as variáveis que demonstraram correlações significativas com as variáveis dependentes (Tabela 2). A ilusão pelo trabalho teve como variáveis preditoras a preocupação empática, o controle sobre o processo de trabalho e o grau de recompensa obtido, sendo essa última a variável com maior peso no modelo preditivo $(\beta=0.35)$ (Tabela 3). O modelo explicou $38 \%$ da variância total da ilusão pelo trabalho $\left(R^{2}=0.38, p<.001\right)$. Angústia pessoal, demanda, apoio social, esforço, recompensa e idade dos participantes foram preditores significativos dos níveis de desgaste psíquico (Tabela 3$)$, sendo a variável esforço $(\beta=0.39)$ a que mais contribuiu para a explicação de $55 \%$ da variância total obtida com o modelo $\left(R^{2}=0.55, p<\right.$ .001). A indolência teve como preditores a preocupação empática, variável de maior peso no modelo $(\beta=-0.36)$, a angústia pessoal e o apoio social $\left(R^{2}=\right.$ $0.31, p<.001)$. Contribuíram significativamente para a explicação de $17 \%\left(R^{2}=0.17, p<.001\right)$ da variância de culpa as variáveis esforço, overcommitment e angústia pessoal, essa última apresentando o maior peso no modelo $(\beta=0.26)$.

O Perfil 1 de Burnout foi identificado em $14.4 \%(n=50)$ da amostra, e o Perfil 2, em $44.5 \%$ $(n=155)$ dos participantes. Portanto, 205 de 348 participantes do estudo apresentaram nível crítico de Burnout (58.9\% da amostra). A Tabela 4 apresenta o modelo final de regressão logística para Perfil 1 e Perfil 2 da síndrome. A variável que foi preditora significativa da ocorrência de Perfil 1 foi recompensa $(O R=0.92)$. Aumentando em uma unidade a recompensa, a chance de ocorrência de Perfil 1 de Burnout diminui em 0.8\%. O modelo obtido explicou $25 \%$ da variância na presença de Perfil 1 da síndrome (Nagelkerke $R^{2}=0.25$ ).

Dentre os participantes que apresentaram Perfil 1 ou Perfil 2 da síndrome, as variáveis que predisseram significativamente a ocorrência de Perfil 2 de Burnout foram angústia pessoal e esforço, tendo o mesmo peso para o modelo $(O R=1.12)$. Aumentando em uma unidade a angústia pessoal ou de esforço, as chances de ocorrência de Perfil 2 de Burnout aumentaram em 1.12 vezes. A explicação da variância obtida com o modelo foi de $14 \%$ (Nagelkerke $R^{2}=0.14$ ). 


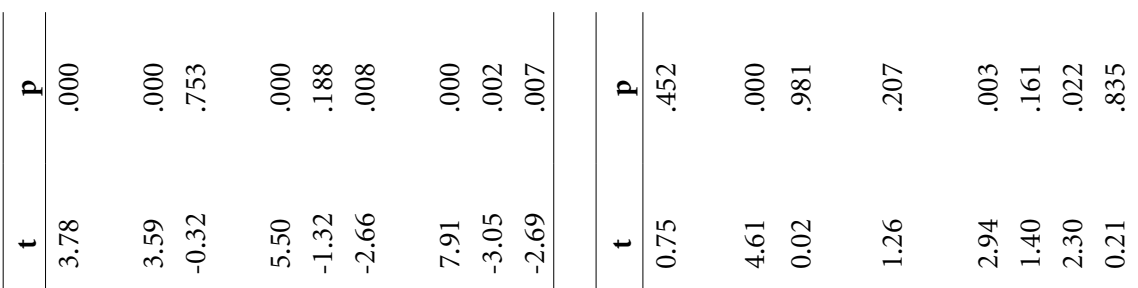

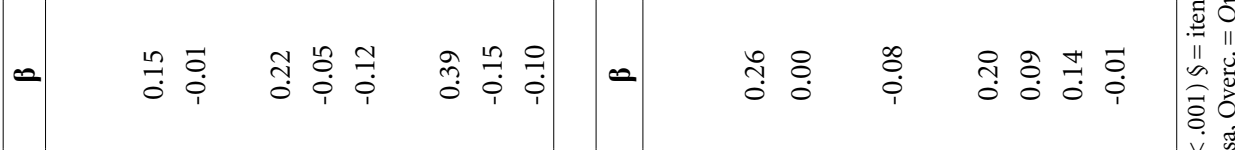

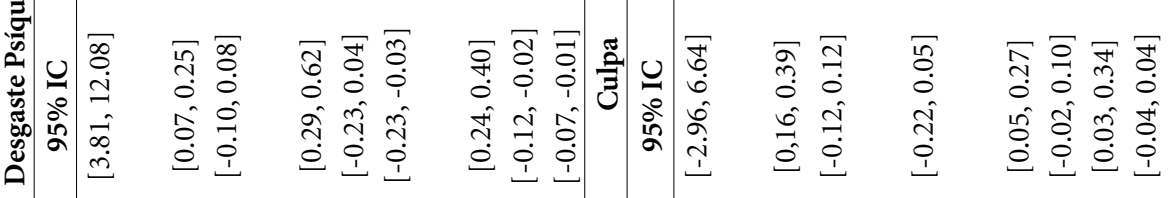

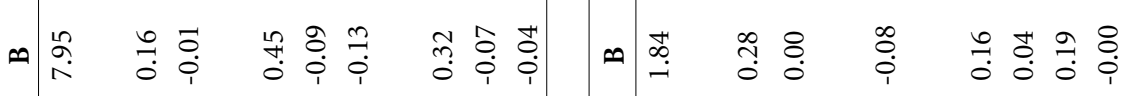

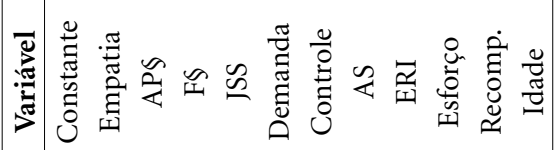

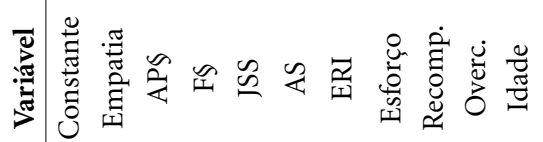

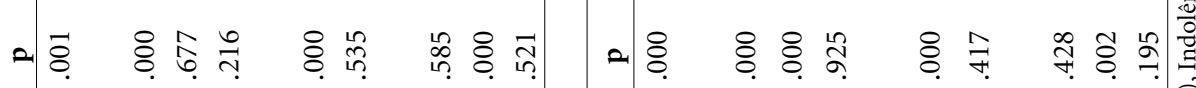

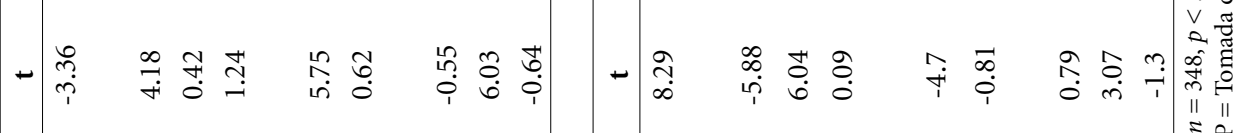

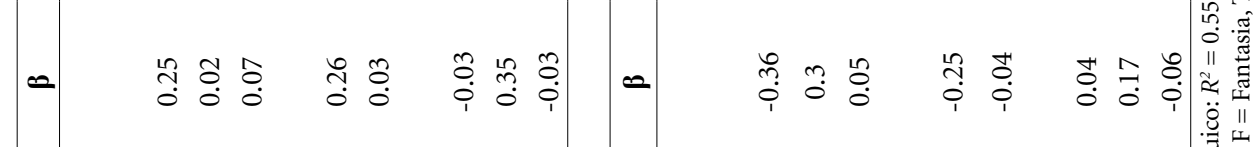

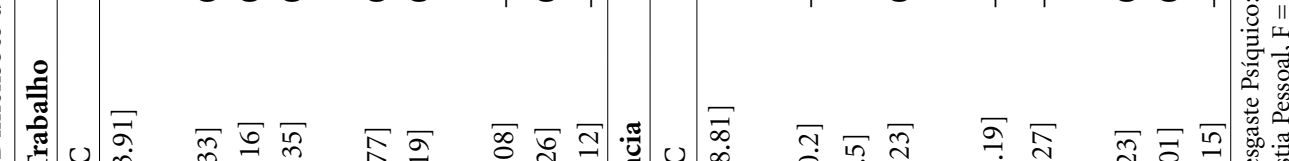

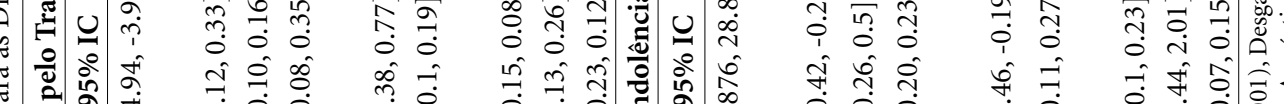

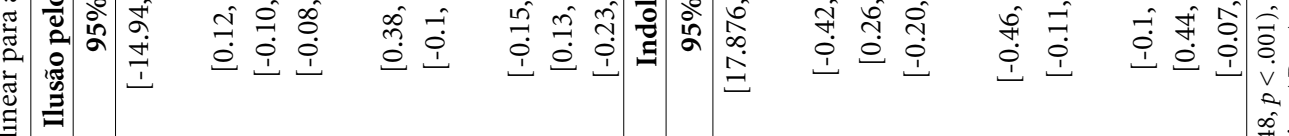

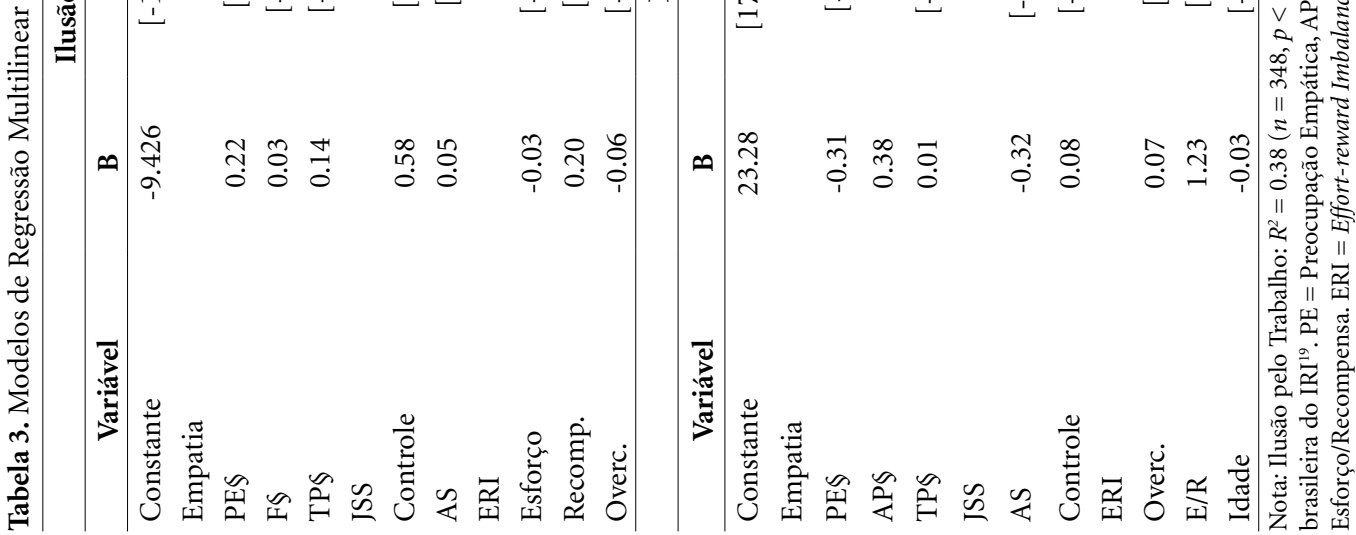


Tabela 4. Modelos de Regressão Logística para a predição dos perfis de Burnout.

\begin{tabular}{lcccccc}
\hline \multicolumn{1}{c}{ Variável } & B & SE & Wald & p & OR & 95\% IC \\
\hline Constante & 4.95 & 2.32 & 4.54 & .033 & 140.78 & \\
JSS & & & & & & \\
Controle & -0.17 & 0.09 & 3.38 & .066 & 0.84 & {$[0.7,1.01]$} \\
AS & -0.07 & 0.07 & 1.08 & .298 & 0.93 & {$[0.82,1.06]$} \\
ERI & & & & & & \\
Esforço & .04 & 0.06 & 0.45 & .504 & 1.04 & {$[0.93,1.16]$} \\
Recomp. & -0.08 & 0.03 & 7.09 & .008 & 0.92 & {$[0.87,0.98]$} \\
Overc. & 0.03 & 0.08 & 0.13 & .716 & 1.03 & {$[0.88,1.2]$} \\
\hline \multicolumn{1}{c}{ Variável } & $\mathbf{B}$ & SE & Wald & $\mathbf{p}$ & $\mathbf{O R}$ & $\mathbf{9 5 \%}$ IC \\
\hline Constante & -3.33 & 1.20 & 7.62 & .006 & 0.04 & \\
Empatia & & & & & & \\
APS & 0.11 & 0.05 & 4.84 & .028 & 1.12 & {$[1.01,1.23]$} \\
ERI & & & & & & \\
Esforço & 0.11 & 0.04 & 6.25 & .012 & 1.12 & {$[1.02,1.22]$} \\
Overc. & 0.07 & 0.07 & 0.90 & .342 & 1.07 & {$[0.93,1.24]$} \\
\hline
\end{tabular}

Nota: Modelo para Perfil 1: $\mathrm{N}=193$, Hosmer-Lemeshow goodness of fit $p=.408,-2 \mathrm{LL}=184.46$, Nagelkerke $R^{2}=0.25$. Modelo para Perfil 2: $\mathrm{N}=205$, Hosmer-Lemeshow goodness of fit $p=.102,-2 \mathrm{LL}=195.99$, Nagelkerke $R^{2}=0.14$. JSS $=$ Job Stress Scale, AS= Apoio Social, ERI $=$ Effort-reward Imbalance, Recomp. $=$ Recompensa, Overc. $=$ Overcommitment, Equip.$=$ disponibilidade de equipamentos para desempenhar função, AP = Angústia Pessoal. $\$=$ itens refatorializados da versão brasileira do IRI ${ }^{19}$.

\section{Discussão}

\section{Relação entre as dimensões de empatia e de Burnout}

A correlação positiva da preocupação empática com a ilusão pelo trabalho e sua relação negativa com a indolência corroboram investigações anteriores com graduandos em medicina ${ }^{29-31}$. Segundo Gleichgerrcht et al. ${ }^{13}$, os efeitos positivos da empatia sobre os profissionais dependeriam da regulação da ativação emocional do trabalhador com o sofrimento do paciente. Essa regulação possibilitaria amenizar certos aspectos da empatia, como a angústia pessoal, e ampliar outros, como a preocupação empática, liberando recursos cognitivos para o manejo de situações clínicas e favorecendo comportamentos de ajuda saudáveis para o profissional, que preveniriam efeitos nocivos do estresse crônico como o Burnout. Halpern ${ }^{32}$ coloca ainda que a sintonia emocional com o paciente e o interesse genuíno por sua situação, aspectos emocionais da empatia relacionados à preocupação empática, atribuiriam maior sentido e satisfação à atuação profissional, o que poderia contribuir para prevenir o Burnout, justificando as correlações averiguadas.

No que tange à angústia pessoal, Thirioux et al. ${ }^{8}$ consideram que essa forma de empatia reduz a experiência alheia a uma perspectiva egocentrada, tendendo a fundir identidades. Esse processo aumentaria a propensão dos profissionais à exaustão emocional, pela atribuição a si dos sentimentos vividos pelos pacientes ${ }^{8}$, o que vai ao encontro da literatura ${ }^{29}$ e da relação positiva entre angústia pessoal e desgaste psíquico encontrada no presente estudo. A correlação entre angústia pessoal e indolência também condiz com uma investigação anterior ${ }^{29}$ e pode estar relacionada com a assoiação entre angústia pessoal e desgaste psíquico, uma vez que, conforme Gil-Monte ${ }^{2}$, o esgotamento emocional crônico contribui ao longo do tempo para a mudança de atitude com os pacientes. No que tange à relação entre angústia pessoal e culpa, ainda que não seja possível inferir causalidade a partir dos resultados, pode-se postular que maiores níveis dessa forma de empatia possam tornar mais aflitivos para os profissionais os sinais de descontentamento de colegas, chefia e usuários em relação ao seu desempenho, desencadeando maiores sentimentos de culpabilização.

A fantasia não tem sido incluída em estudos sobre a relação entre empatia e Burnout que utilizem o IRI ${ }^{13,29-31}$, o que impossibilita comparações dos resultados apresentados aqui com a literatura. Todavia, essa dimensão de empatia apresentou apenas correlações muito fracas ${ }^{23}$ com o Burnout, 
tanto inversa (ilusão pelo trabalho) quanto diretamente (desgaste psíquico e culpa) relacionadas aos sintomas da síndrome. Os resultados sugerem papel indefinido ou pouco relevante dessa dimensão na associação da empatia com o Burnout.

A correlação negativa fraca da tomada de perspectiva com a ilusão pelo trabalho apresentada nesse estudo é condizente com os achados de Paro et al. ${ }^{29}$ com estudantes de medicina, assim como a correlação fraca dessa dimensão de empatia com a indolência. Segundo Thirioux et al. ${ }^{8}$, a divisão entre o eu e o outro e a consideração por sua perspectiva pessoal e alteridade, característicos da tomada de perspectiva, justificariam a prevenção da exaustão emocional e da despersonalização ${ }^{8}$ (dimensão análoga à indolência no modelo de Gil-monte ${ }^{2}$ ). Por outro lado, o resultado encontrado no presente estudo contraria o achado de Tei et al..$^{33}$, que relaciona positivamente tomada de perspectiva e exaustão emocional em enfermeiros.

As correlações obtidas sugerem que a relação entre empatia e Burnout varia drasticamente dependendo da forma de empatia aferida. $\mathrm{O}$ presente estudo, ainda que não englobe a diversidade de medidas de empatia da literatura, traz resultados que corroboram a hipótese de Zenasni et al. ${ }^{14}$ de que a variedade de definições e medidas de empatia pode ser a causa subjacente da falta de unanimidade na literatura a respeito da associação entre empatia e Burnout.

\section{Comparação entre as associações da empatia e do estresse ocupacional com as dimensões e perfis de Burnout}

As associações encontradas entre os fatores de proteção contra o estresse ocupacional, controle, recompensa e apoio social, e os menores níveis de Burnout corroboram estudos anteriores com profissionais de saúde ${ }^{34-41}$. Da mesma forma, a associação da demanda e do esforço com maiores níveis de Burnout entre profissionais de saúde condiz com a literatura ${ }^{34,37,39-44}$. Por fim, de acordo com o postulado por Siegrist ${ }^{7}$ de que o estado de estresse do profissional poderia ser ampliado pelo comprometimento exagerado com as tarefas, o overcommitment aparece diretamente correlacionado com o Burnout. É possível que esse tenha elevado a intolerância dos participantes em relação ao seu mau desempenho laboral, o que poderia justificar a predição significativa do overcommitment sobre a culpa.

Ainda que variáveis ligadas ao estresse ocupacional tenham apresentado as maiores correla- ções com o Burnout, houve alternância entre essas e a empatia como principais preditores a depender da dimensão da síndrome tomada como variável dependente. Os resultados obtidos sugerem uma maior associação de variáveis relacionadas ao estresse ocupacional com as dimensões de Burnout que são consideradas por Gil-Monte ${ }^{2}$ as precursoras da síndrome. O primeiro sintoma cognitivo de Burout seria o rebaixamento da ilusão pelo trabalho, que teve como principal preditor a percepção de não estar sendo devidamente reconhecido e recompensado pelo trabalho (recompensa). Afetivamente, o Burnout inicia com o desgaste psíquico ${ }^{2}$, que por sua vez mostrou-se principalmente associado à percepção do esforço requerido frente à carga de trabalho e sua complexidade.

Por outro lado, os resultados encontrados apontam que a maior influência da empatia poderia ocorrer em um estágio mais tardio do Burnout, quando surgem a indolência e a culpa. A indolência consistiria em uma estratégia de enfrentamento diante dos estados de desilusão e exaustão emocional crônicos, através de mudança atitudinal e de estabelecimento de conduta negativa frente às pessoas atendidas, e que podem culminar em sentimentos de culpa pelo proceder inadequado do profissional ${ }^{2}$. Seria possível que, apesar de estabelecidos os sintomas iniciais da síndrome, a preocupação empática prevenisse o desenvolvimento de atitude negativa em relação aos usuários, enquanto a angústia pessoal favoreceria os sentimentos de culpabilização em sua decorrência. Contudo, tratando-se de um estudo transversal, as inferências de causalidade entre as variáveis precisam ser tomadas com cuidado.

No que tange aos perfis de Burnout, as percentagens de Perfil 1 (14.4\%) e Perfil 2 (44.5\%) foram consideravelmente mais elevadas do que aquelas encontradas por Silveira et al. ${ }^{45}$ entre profissionais da APS, com $11 \%$ de Perfil 1 e $18 \%$ de Perfil 2, apesar das pesquisas terem sido realizadas em um mesmo município e usando o mesmo instrumento. Ainda que a amostragem tenha sido não probabilística em ambos os estudos, essa diferença pode ser atribuída por diferenças amostrais, uma vez que a investigação de Silveira et $a l .{ }^{45}$ se restringiu a dois distritos sanitários, enquanto no presente estudo participaram profissionais dos oito distritos do município. Além disso, a forma de levantamento da escala foi ligeiramente diferente entre os dois estudos. Silveira et al. ${ }^{45}$ tomaram como ponto de corte o escore médio 2 para a obtenção do perfis de Burnout considerando a pontuação de cada dimensão da 
escala separadamente. Também utilizando como ponto de corte o escore médio 2, Carlotto et al. ${ }^{46}$ encontraram entre professores de ensino especial prevalência semelhante à de Silveira et al ${ }^{45}$ para Perfil 2, de 14.3\%, enquanto para Perfil 1 obtiveram $30.6 \%$.

No presente estudo, considerou-se ponto de corte para a pontuação total $(20$ - It + Dp + In $)$ e para culpa o percentil 90 da amostra de referência, usando-se os escores médios apresentados por Gil-Monte ${ }^{2}$ a partir de amostra de 3320 profissionais de saúde de diferentes países (pontuação total $/ 15 \geq 1.93$, média dos itens de culpa $\geq 1.8$ ). Estudos utilizando o percentil 90 de sua própria amostra como ponto de corte encontraram prevalências bastante distintas daquelas apresentadas no presente estudo, porém semelhantes entre si. Entre bombeiros ${ }^{25}, 9.5 \%$ de Perfil 1 e $3.5 \%$ de Perfil 2, e entre gestores municipais de saúde ${ }^{26}, 3.0 \%$ de Perfil 1 e $2.3 \%$ de Perfil 2 .

Dessa forma, as diferenças entre as percentagens de Burnout obtidas na presente investigação e as Silveira et al. ${ }^{45}$ podem ser atribuídas a diferenças metodológicas, ainda que não possa ser descartada uma piora dos sintomas de Burnout entre os profissionais do municipio ao longo do intervalo entre as investigações. De qualquer forma, é preocupante que mais da metade dos profissionais acessados (59\%, somando os perfis) tenham exibido um grau elevado de comprometimento pelo Burnout quando comparados à amostra normativa ${ }^{2}$, e que desses a maioria apresente níveis elevados de culpa pelo seu desempenho no trabalho, o que caracteriza indício da forma mais grave da síndrome ${ }^{2}$.

Da mesma forma que os modelos de regressão linear para as dimensões de Burnout, os preditores obtidos para seus perfis sugerem maior contribuição do estresse ocupacional (recompensa) para o alcance dos primeiros patamares de gravidade da síndrome (Perfil 1), assim como uma contribuição crescente da empatia ao longo do desenvolvimento do Burnout, tendo a angústia pessoal peso equivalente ao esforço na predição da forma mais grave da síndrome (Perfil 2).

Como se poderia esperar, as variáveis que se mostram significativamente relevantes para a predição da ocorrência de Perfil 2 assemelhamse àquelas que compõem as preditoras de culpa, uma vez que os níveis dessa dimensão diferenciam os dois perfis. Contudo, na regressão multilinear para esse sintoma de Burnout, o overcommitment contribuiu significativamente para o modelo e o peso da angústia pessoal superou o do esforço. Considerando que o ponto de corte esta- belecido a partir de amostra de referência ${ }^{2}$ sugere que quase metade dos respondentes $(44,5 \%)$ apresentaram o Perfil 2 de Burnout, é possível que as características individuais (overcommitment $\mathrm{e}$ angústia pessoal) sejam de maior relevância para estabelecer níveis de culpabilização que superem o patamar necessário para Perfil 2, culminando, portanto, em manifestações ainda mais graves da síndrome.

\section{Limitações do estudo}

É oportuno revelar algumas limitações deste trabalho. Como qualquer estudo com amostragem não probabilística, a generalização dos resultados obtidos deve ser considerada com cautela. O estudo utilizou medidas de autorrelato, o que torna os resultados suscetíveis à desejabilidade social, porém na atualidade não se dispõem de outros métodos para a avaliação destas variáveis. Por outra parte, a versão brasileira do questionário CESQT, que mede a variável de resultado principal no presente estudo, inicialmente validada no Brasil com uma amostra de professores, e posteriormente utilizada em estudos com profissionais da $\mathrm{APS}^{45}$, bombeiros ${ }^{25}$, e gestores de saúde $^{26}$, ainda não conta com normas para a população brasileira, o que traz dificuldades na segurança quanto a classificação dos participantes nos possíveis níveis ou perfis de Burnout.

Ainda, a presente investigação aplicou um delineamento transversal, o que restringe a possibilidade de inferir causalidade a partir das associações averiguadas entre as variáveis estudadas. Por último, uma falha na coleta impediu o uso de todos os itens do Interpersonal Reactivity Index. Embora tenha sido mantida a coerência teórica entre items e fatores, e similaridade na estrutura fatorial do instrumento original e a versão fatorializada para esse estudo, é possível que exista algum viés nos resultados obtidos envolvendo as dimensões de empatia de Davis' ${ }^{9}$, por conta da não utilização da escala completa (perda de 6 items).

\section{Considerações finais}

Os resultados desse estudo indicam a importância de se considerar as diferentes formas de empatia para determinar a sua relação com o Burnout, sugerindo que atomada de perspectiva e, em especial, apreocupação empática possam ser fatores protetivos ao Burnout, enquanto a angústia pessoal apresenta-se como potencial fator de risco. $\mathrm{O}$ estudo também permitiu inferir 
que variáveis relacionadas ao estresse ocupacional possam contribuir mais fortemente para as dimensões precursoras do Burnout enquanto a empatia teria papel mais importante para o estabelecimento dos casos mais extremos da síndrome. Esses resultados sugerem que intervenções de combate ao Burnout com profissionais da APS deveriam enfocar tanto os recursos e estressores presentes no ambiente de trabalho quanto a regulação e refinamento das habilidades empáticas dos trabalhadores. Espera-se que esse estudo, somado ao corpo de evidências de fatores associados ao Burnout na APS brasileira, ajude a embasar as providências para o enfrentamento da síndrome e para a garantia do direito à saúde de trabalhadores e usuários.

\section{Colaboradores}

JP Pinheiro concebeu a pesquisa, realizou a coleta, análise e interpretação dos dados e a redação do manuscrito. JB Sbicigo colaborou com as análises estatísticas, com a revisão crítica do artigo e com a aprovação da versão a ser publicada. E Remor colaborou com a concepção da pesquisa, com as análises estatísticas, com a revisão crítica do artigo e com a aprovação da versão a ser publicada.

\section{Referências}

1. Maslach C, Jackson SE. The Measurement of Experienced Burnout. J Organizational Behav 1981; 2(2):99113.

2. Gil-Monte PR. Cuestionario para la Evaluación del Síndrome de Quemarse por el Trabajo - Manual. Madri: TEA Ediciones; 2011.

3. Gil-Monte PR, Carlotto MS, Câmara SG. Validação da versão brasileira do "Cuestionario para la Evaluación del Síndrome de Quemarse por el Trabajo” em professores. Rev Saude Publica 2010; 44(1):140-147.

4. Aronsson G, Theorell T, Grape T, Hammarström A, Hogstedt C, Marteinsdottir I, Skoog I, Träskman-Bendz L, Hall C. A systematic review including meta-analysis of work environment and burnout symptoms. BMC Public Health 2017; 17(1):264.

5. Theorell T, Karasek RA. Current issues relating to psychosocial job strain and cardiovascular disease research. J Occup Health Psychol 1996; 1(1):9-26.

6. Siegrist J. Adverse health effects of high-effort/ low-reward conditions. J Occup Health Psychol 1996; 1(1):27-41.

7. Pereira DG. Síndrome de Burnout em trabalhadores do Programa de Saúde da Família: uma revisão de literatura [monografia]. Belo Horizonte: Universidade Federal de Minas Gerais; 2011.

8. Thirioux B, Birault F, Jaafari N. Empathy Is a Protective Factor of Burnout in Physicians: New Hypotheses Regarding Empathy and Sympathy in Care Relationship. Front Psychol 2016; 7:763.

9. Davis $\mathrm{MH}$. Measuring individual differences in empathy: evidence for a multidimensional approach. $J$ Personality Social Psychol 1983; 44(1):113-136.

10. Figley CR. Compassion Fatigue: Psychotherapists' Chronic Lack of Self Care. J Clin Psychol 2002; 58(11):1433-1441.

11. Ferri P, Guerra E, Marcheselli L, Cunico L, Di Lorenzo R. Empathy and burnout: an analytic cross-sectional study among nurses and nursing students. Acta Biomed 2015; 86(2):104-115.

12. Park C, Lee YJ, Hong M, Jung C, Synn Y, Kwack Y, Ryu J, Park T, Lee S, Bahn G. A Multicenter Study Investigating Empathy and Burnout Characteristics in Medical Residents with Various Specialties. J Korean Med Sci 2016; 31(4):590-597. 
13. Gleichgerrcht E, Decety J. Empathy in Clinical Practice: How Individual Dispositions, Gender, and Experience Moderate Empathic Concern, Burnout, and Emotional Distress in Physicians. PLoS One 2013; 8(4):e61526.

14. Zenasni F, Boujut E, Woerner A, Sultan S. Editorials Burnout and empathy in primary care: three hypothesis. Br J Gen Pract 2012; 62(600):346-347.

15. Shaughnessy J, Zechmeister E, Zechmeister J. Research Methods in Psychology. New York: McGraw-Hill; 2009.

16. Osorio RG. A classificação de cor ou raça do IBGE revisitada. In: Petrucelli JL, Saboia AL, organizadores. Características Étnico-Raciais da População, Classificações e Identidades. Rio de Janeiro: IBGE; 2013. p. 83-99.

17. Sampaio LR, Guimarães PRB, Camino CPS, Formiga NS, Menezes IG. Estudos sobre a dimensionalidade da empatia: tradução e adaptação do Interpersonal Reactivity Index (IRI). Psico PUCRS 2011; 42(1):67-76.

18. Izquierdo I, Olea J, Abad FJ. Exploratory factor analysis in validation studies: uses and recommendations. Psicothema 2014; 26(3):395-400.

19. Alves MGDM, Chor D, Faerstein E, Lopes CDS, Werneck GL. Short version of the "job stress scale": a Portuguese-language adaptation. Rev Saude Publica 2004; 38(2):164-171.

20. Theorell T. Working conditions and health. In: Berkman L, Kawachi I, editores. Social epidemiology. New York: Oxford University Press; 2000. p. 95-118.

21. Maslach C, Leiter MP. Early Predictors of Job Burnout and Engagement. J Applied Psychol 2008; 93(3):498512.

22. Le Blanc PM, Hox JJ, Schaufeli WB, Taris TW, Peeters MCW. Take Care! The Evaluation of a Team-Based Burnout Intervention Program for Oncology Care Providers. J Applied Psychol 2007; 92(1):213-227.

23. Siegrist J, Starke D, Chandola T, Godin I, Marmot $\mathrm{M}$, Niedhammer I, Peter R. The measurement of effort-reward imbalance at work: European comparisons. Soc Sci Med 2004; 58(8):1483-1499.

24. Chor D, Werneck GL, Faerstein E, Alves, MGDM, Rotenberg L. The Brazilian version of the effort-reward imbalance questionnaire to assess job stress. $\mathrm{Cad}$ Saúde Pública 2008; 24(1):219-224.

25. Melo LP, Carlotto MS. Prevalência e Preditores de Burnout em Bombeiros. Psicol Cien Prof 2016; 36(3):668-681.

26. Poletto NA, Probst LF, Oliveira TL, Guerra LM, Ambrosano MB, Cortellazzi KL, Gil-Monte PR, Possobon RF. Síndrome de Burnout em gestores municipais da saúde. Cad Saúde Colet 2016; 24(2):209-215.

27. Dattoli AR, Pérez RCG, Silva MI, González MC. El síndrome de quemarse por el trabajo y factores psicosociales en docentes de primaria de la ciudad de montevideo. Cien Psicol 2015; 9(2):273-281.

28. Dancey CP, Reidy J. Estatística sem matemática para psicologia: usando SPSS para Windows. $3^{\text {a }}$ ed. Porto Alegre: Artmed; 2006.

29. Paro HBMS, Silveira PSP, Perotta B, Gannam S, Enns SC, Giaxa RRB, Bonito RF, Martins MA, Tempski PZ. Empathy among medical students: Is there a relation with quality of life and burnout? PLoS One 2014; 9(4):e94133.

30. Thomas MR, Dyrbye LN, Huntington JL, Lawson KL, Novotny PJ, Sloan JA. How Do Distress and Well-being Relate to Medical Student Empathy? A Multicenter Study. J Gen Intern Med 2007; 22(2):177-183.
31. von Harscher H, Desmarais N, Dollinger R, Grossman S, Aldana $S$. The impact of empathy on burnout in medical students: new findings. Psychol Health Med 2017; 23(3):295-303.

32. Halpern J. What is Clinical Empathy? J Gen Intern Med 2003; 18(8):670-674.

33. Tei S, Becker C, Kawada R, Fujino J, Jankowski KF, Sugihara G, Murai T, Takahashi H. Can we predict burnout severity from empathy-related brain activity? Transl Psychiatry 2014; 4(6):e393.

34. Bougea A, Kleisarchakis MK, Spantideas N, Voskou P, Thomaides T, Chrousos G, Belegri SA. Job stress and burnout in the care staff of Leros PIKPA Asylum 25 years after the first Deinstitutionalisation and Rehabilitation Greek Project. BJPsych Bull 2016; 40(6):296301.

35. Day A, Crown SN, Ivany M. Organisational change and employee burnout: The moderating effects of support and job control. Safety Sci 2017; 100(A):4-12.

36. Gasparino RC, Guirardello EB, Aiken LH. Validation of the Brazilian version of the Nursing Work Index-Revised. J Clinical Nurs 2011; 20(23-24):3494-3501.

37. Jourdain G, Chênevert, D. Job demands-resources, burnout and intention to leave the nursing profession: A questionnaire survey. Int J Nurs Studies 2010; 47(6):709-722.

38. Lorenz VR, Guirardello EB. O ambiente da prática profissional e Burnout em enfermeiros na atenção básica. Rev Lat-Ame Enferm 2004; 22(6):926-933.

39. Sundin L, Hochwälder J, Lisspers J. A longitudinal examination of generic and occupational specific job demands, and work-related social support associated with burnout among nurses in Sweden. Work 2011; 38(4):389-400.

40. Vandenbroeck S, Van Gerven E, De Witte H, Vanhaech $\mathrm{K}$, Godderis L. Burnout in Belgian physicians and nurses. Occup Med 2017; 67(7):546-554.

41. Verweij $H$, van der Heijden, FMMA, van Hooff MLM, Prins JT, Lagro-Janssen ALM, van Ravesteijn $\mathrm{H}$, Speckens AEM. The contribution of work characteristics, home characteristics and gender to burnout in medical residents. Adv Health Sci Educ Theory Pract 2017; 22(4):803-818.

42. Bakker $\mathrm{AB}$, Killmer $\mathrm{CH}$, Schaufeli WB. Effort-reward imbalance and burnout among nurses. J Adv Nurs 2000; 31(4):884-891.

43. Luan X, Wang P, Hou W, Chen L, Fenglan L. Job stress and burnout: A comparative study of senior and head nurses in China. Nurs Health Sci 2017; 19:163-169.

44. Torppa MA, Kuikka L, Nevalainen M, Pitkälä KH. Emotionally exhausting factors in general practitioners' work. Scand J Prim Health Care 2018; 33(3):178-183.

45. Silveira S, Câmara S, Amazarray MR. Burnout predictors in health professionals of primary care of Porto Alegre/RS, Brazil. Cad Saúde Colet 2014; 22(4):386392.

46. Carlotto MS, Librelotto R, Pizzinato A, Barcinski M. Prevalência e factores associados à Síndrome de Burnout nos professores de ensino especial. Análise Psicol 2012; 30(3):315-327.

Artigo apresentado em 24/07/2018

Aprovado em 18/12/2018

Versão final apresentada em 20/12/2018 